\title{
Vorwort zur 16. Auflage
}

Bei der wissenschaftlichen und praktischen Arbeit mit bürgerlichen Rechtsordnungen in Deutschland und Europa stehen Lehrbücher, Fachzeitschriften und Tagungsbeiträge gewöhnlich neben der einschlägigen, besonders in Deutschland sehr starken Kommentar-Literatur. Deren Verfasser sind daher gehalten, bei der Darstellung von und Stellungnahme zu praktisch wie wissenschaftlich wichtigen Einzelfragen stets die Problemsichten in den Mittelpunkt zu stellen, die sich auf der Grundlage der einschlägigen Kodifikation mit ihrer Dogmatik und der darin verarbeiteten gesellschaftlichen Vorstellungen entwickelt haben. Dies darf jedoch die Praktikabilität von Einzelregeln und -lösungen nicht beeinträchtigen, führt aber dazu, dass bei der Kommentierung einer Vorschrift sehr häufig deutlich über den reinen Gesetzestext hinauszugehen ist und Lösungen und Meinungen immer mit den Grundlagen und Hintergründen der Norm abzustimmen sind. Das trägt zum Umfang der Kommentierungen bei. Der hiermit in 16. Auflage vorgelegte Kommentar zum BGB und seinen wichtigsten Nebengesetzen hat von seinem ersten Erscheinen an das Ziel verfolgt, durch die Zusammenarbeit von in Wissenschaft und Praxis ausgewiesenen Autoren den beschriebenen Anforderungen gerecht zu werden. Ziel der Autoren ist es, auf der Grundlage ihrer praktischen Erfahrungen und Vertrautheit mit wissenschaftlicher Arbeit diese Ansprüche so zu erfüllen, so dass trotz einer großen Zahl von Fachleuten eine geschlossene Kommentierung entsteht.

Mit der 16. Auflage befindet sich der Kommentar auf dem Gesetzesstand vom 1.4.2020. Berücksichtigt sind das Gesetz zur Abmilderung der Folgen der COVID-19 Pandemie im Zivil-, Insolvenz- und Strafverfahrensrecht, das Gesetz zur Umsetzung des Gesetzes zur Einführung des Rechts auf Eheschließung für Personen gleichen Geschlechts, das Gesetz zur Umsetzung der RL (EU) 2016/680 im Strafverfahren sowie zur Anpassung datenschutzrechtlicher Bestimmungen an die VO (EU) 2016/679, das Gesetz zur Verlängerung und Verbesserung der Regelungen über die zulässige Miethöhe bei Mietbeginn, das Gesetz zur Verlängerung des Betrachtungszeitraums für die ortsübliche Vergleichsmiete, das Gesetz zur Förderung der Freizügigkeit von EU-Bürgerinnen und -Bürgern sowie zur Neuregelung verschiedener Aspekte des Internationalen Adoptionsrechts, das Gesetz zur Anpassung der Betreuer- und Vormündervergütung sowie das Gesetz zur Umsetzung der Entscheidung des Bundesverfassungsgerichts vom 26. März 2019 zum Ausschluss der Stiefkindadoption in nichtehelichen Familien. Zudem hat Herr Professor Herbert Grziwotz die anstehende Reform des Wohnungseigentumsrechts in seiner Kommentierung bereits berücksichtigt und gibt einen Ausblick auf die zu erwartenden Änderungen. Seit der Vorauflage hat es auch wieder eine Menge wichtiger höchstrichterlicher und instanzgerichtlicher Rechtsprechung gegeben. Hier die richtige Auswahl zu treffen, die wesentlichen Aussagen in den Sachzusammenhang zu stellen und die Folgen zu beleuchten, ist ebenfalls seit jeher ein Verdienst des Erman. Mit punktuellen Online-Aktualisierungen an Ort und Stelle wird der Erman zudem in der Datenbank auch zwischen den Auflagen aktuell gehalten.

Die mit einem Werk dieses Zuschnitts verbundenen Ansprüche an die fachliche Spezialisierung und Arbeitskraft der Autoren und der Wunsch nach altersbedingtem Ausscheiden haben auch in dieser Auflage zu Wechseln in der Autorenschaft und einer weiteren Vergrößerung des Bearbeiterkreises geführt. So ist der Mitherausgeber Dr. Georg Maier-Reimer aus der Autorenschaft ausgeschieden und hat die $\$ \$ 164-193$ an Professor Dr. Thomas Finkenauer übergeben. Die Kommentierung des AGB-Rechts ist von Ri'inBGH Dr. Stefanie Roloff auf Prof. Dirk Looschelders übergegangen. Dr. Andreas Dieckmann hat die $\$ \$ 311-311$ a von Prof. Dr. Johann Kindl und Prof. Dr. Axel Metzger die \$\$336-354 von Prof. Dr. Stefan Schaub und Prof. Dr. Anne Röthel übernommen. Das bis zur 15. Auflage von Prof. Dr. Ronald Schmid verantwortete Reisevertragsrecht wurde infolge der Umsetzung der neuen Pauschalreise-Richtlinie neu gefasst und wird kommentiert von RiAG Dr. Daniel Blankenburg und RiAG Martin Strube. Neu eingetreten sind des Weiteren RiKG Dr. Oliver Elzer und Dr. Mareike Preisner, die die $\$ \$ 854-872$ und 907-924 (Dr. Elzer) von Dr. Arndt Lorenz und die $\$ \$ 1564-1568 \mathrm{~b}$ (Dr. Preisner) von Klaus-Peter Blank übernommen haben. Ausgeschieden aus dem Autorenkreis ist auch Prof. Dr. Stefan Saar, dessen Kommentierung des Adoptionsrechts von DirAG Dr. Stephan Teklote weitergeführt wird. Schließlich hat Prof. Dr. Harm Peter Westermann seine Bearbeitung im allgemeinen Schuldrecht in jüngere Hände gelegt, in dem jetzt Prof. Dr. Sebastian Martens die Einleitung zu $\$ 241$ und $\$ 241$ sowie die $\$ \$ 398-413$ und Prof. Dr. Daniel Ulber die Kommentierung der $\$ \$ 243,275-285,320-327$ verantworten. Zudem hat Prof. Dr. Walter Bayer zusätzlich zu seinen Kommentierungen im Sachen- und Erbrecht die Kommentierung der $\$ \$ 328-335$ übernommen.

Die Kommentierung des Internationalen Privatrechts ist von Prof. Dr. Gerhard Hohloch auf Prof. Dr. Michael Stürner und Dr. Adrian Hemler übergegangen. Dabei wurde der Aufbau modifiziert und die Kommentierung des EGBGB vorangestellt; im Anschluss folgt die Kommentierung der einschlägigen EU-Verordnungen (Verordnungen Rom I, II und III, EuGüVO, EuPartVO, EuUnthVO, Haager Unterhaltsprotokoll und EuErbVO).

Den Ausgeschiedenen gebührt für ihre umfangreichen, wissenschaftlich wie praktisch bedeutenden Beiträge der Dank des gesamten Mitarbeiterkreises und besonders der Herausgeber. Ein besonderer Dank der Herausgeber gilt den Herren Professor Hohloch und Professor Saar, die den Kommentar über viele Auflagen begleitet und durch ihre umsichtigen Ausführungen bereichert haben. Die Herausgeber freuen sich, bei dieser Gelegenheit einen ausdrücklichen Dank auch der umsichtigen und sachkundigen verlegerischen Betreuung durch Frau Sonja BehrensKhaled aussprechen zu können. Dank gebührt auch Frau Friederike Voss für tatkräftige Unterstützung im Lektorat und Frau Heike Tillenburg für die Erstellung des Stichwortverzeichnisses. 
Hinweise und Anregungen, die dazu beitragen, das hohe Niveau des Kommentars zu halten und weiter zu verbessern, können unter lektorat@otto-schmidt.de direkt an den Verlag gerichtet werden.

Köln und Tübingen, im Juli 2020

Barbara Grunewald

Georg Maier-Reimer

Harm Peter Westermann 